\title{
Azathioprine Treatment in Systemic Lupus Erythematosus: A Double Edged Sword
}

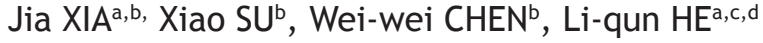 \\ ${ }^{a}$ Nephrology Department, Shuguang Hospital affiliated to Shanghai University of Traditional Chinese Medicine. \\ ${ }^{b}$ Rheumatology Department, Shanghai Municipal Hospital of Traditional Chinese Medicine Affiliated to Shanghai \\ University of Traditional Chinese Medicine \\ 'Key laboratory of Clinical Study For Traditional Chinese Medicine of Shanghai, Key Laboratory of Liver and \\ Kidney Diseases Administrated by The Ministry Of Education \\ ${ }^{d} E$-institute of Chinese Internal Medicine of Shanghai Colleges and Universities
}

\begin{abstract}
Extremely severe pancytopenia induced by low dosage of azathioprine in systemic lupus erythematosus patients is rare. A 40-year-old Chinese female was diagnosed with systemic lupus erythematosus. She suffered worse erythema, oral ulceration, raised erythrocyte sedimentation rate and high anti-dsDNA in August 2013. Then she was initiated on oral azathioprine $50 \mathrm{mg} / \mathrm{d}$ and extremely severe pancytopenia was seen in September 2013. She was recovered by a series of treatments. Regular monitoring of blood counts is highly recommended to reduce the possible serious myelosuppression induced by azathioprine.
\end{abstract}

KEYWORDS: Systemic lupus erythematosus, azathioprine, pancytopenia

\section{CASE REPORT}

A 40-year-old Chinese female was diagnosed with systemic lupus erythematosus due to fever, swollen and painful joints, facial erythema, positive ANA and antidsDNA. She was only treated with oral prednisone and hydroxychloroquine and maintained symptom free in a long time. She suffered worse erythema, oral ulceration, raised erythrocyte sedimentation rate and high antidsDNA in August 2013. Then she was initiated on oral azathioprine $50 \mathrm{mg} / \mathrm{d}$. Her blood counts and function of liver and kidney were unremarkable after 2 weeks of AZA treatment. However, she was admitted to hospital for 3-day high fever coupled with more severe oral ulceration in September the same year. Her complete blood count was as follows: white blood cell count $0.45 \times 10^{9} / \mathrm{L}$ (normal level $4-10 \times 10^{9} / \mathrm{L}$ ), with $0.03 \times 10^{9} / \mathrm{L}$ neutrophils and $0.41 \times 10^{9} / \mathrm{L}$ lymphocytes, haemoglobin $71 \mathrm{~g} / \mathrm{L}$ (normal level $110-160 \mathrm{~g} / \mathrm{L}$ ), erythrocyte count $2.44 \times 10^{12} / \mathrm{L}$ (normal level $3.5-5.5 \times 10^{12} / \mathrm{L}$ ), platelet count $10 \times 10^{9} / \mathrm{L}$ (normal level 100-300 $\times 109 /$ L), suggesting extremely severe pancytopenia. Bone marrow report showed the number of nucleated cells reduced, neutrophils significantly decreased,

Corresponding author:

Li-qun HE

Shuguang hospital affiliated to shanghai university of traditional chinese medicine

No. 145 Pu An Road,

Shanghai postcode 200021

Telephone:20256666

Email:heliqun59@163.com no orthochromatic erythroblast in classification, the number of megakaryocytes declined and rare platelets. Complements, immunoglobulins, anti-dsDNA and urine examination did not reveal a flare of SLE activity. AZA was discontinued and she was prescribed broad-spectrum antibiotics for febrile neutropenia, subcutaneous injection of GM-CSF and Tpo, intravenous human gamma globulin, and received packed platelets. Going through over 3 weeks, her blood counts gradually increased.

\section{DISCUSSION}

Azathioprine as an immunosuppressant is widely prescribed in a lot of autoimmune diseases like systemic lupus nephritis and inflammatory bowel disease(IBD). Its clinical efficacy is well acknowledged but we should still be alert on its adverse drug reaction. In the case, the blood test was normal after two weeks when AZA was taken daily. Nevertheless, extremely severe bone marrow suppression was confirmed over one month despite of the moderate dosage, leading to pancytopenia.

Thiopurine S-methyltransferase (TPMT) is a genetically moderated important enzyme involved in the metabolism of AZA. Deficiency of TPMT activity or its low activity increases AZA toxicity and gives a rise of adverse drug events, among which severe myelosuppression could be fatal. A total of 21 TPMT genetic polymorphisms have been identified which are, or may be associated with decreased levels of TPMT enzyme activity and/or thiopurine drug-induced toxicity. ${ }^{1}$ There is quite difference in TPMT genetic 
polymorphism between Chinese people and European people. Wu et al found no TPMT * 2, TPMT * $3 \mathrm{~A}$ and TPMT * $3 \mathrm{~B}$ in the detection of TPMT ${ }^{*},{ }^{*} 3 \mathrm{~A},{ }^{*} 3 \mathrm{~B}$ and ${ }^{*} 3 \mathrm{C}$ in 150 cases of renal transplantation patients, but 7 cases of TPMT * $3 \mathrm{C}$ mutant heterozygous TPMT alleles. He concluded TPMT * $3 \mathrm{C}$ may be the most important TPMT gene mutation type in the Chinese population. ${ }^{2}$ It seems phenotyping and genotyping of TPMT should be made prior to AZA usage in consideration of drug safety. But enzymatic activity of TPMT could also be influenced by difference in the age of red blood cells ${ }^{3}$ or other oral drugs. A systematic literature review ${ }^{4}$ was performed to assess the reliability of data on costs of AZA-induced neutropenia and performing TPMT pharmacogenetic testing in Europe and it revealed the cost was rather high(the net cost per prevented case of neutropenia equaled to 5,300 euros), which was not a small expenditure.

Besides TPMT, inosine triphosphate pyrophosphatase (ITPA) genes have association with AZA toxicity. Genotypic analysis ${ }^{5}$ showed that there was a statistical significance between c.94C >A variant on ITPA gene with no response to AZA treatment and arthralgia, as well as between mutant TPMT alleles and myelosuppression. Under AZA therapy, ITPA deficiency presumably leads to accumulation of unusual thioinosine metabolite and genotyping of ITPA may be useful to achieve dose optimization. ${ }^{6}$ Japanese patients require lower doses of AZA compared with Caucasian patients to achieve the same concentration of active metabolites and the author suggests the dose of $A Z A<1.5 \mathrm{mg} / \mathrm{kg} /$ day for Asian patients with ITPA 94A allele. ${ }^{6}$ Otherwise, a significant association between inosine triphosphatase IVS2+21A-->C variants with thrombocytopenia was also detected. $^{7}$

Another enzyme is also believed in connection with adverse drug reactions to azathioprine. Stocco et $\mathrm{al}^{8}$ have recently reported that IBD patients with a wildtype glutathione-S-transferase-M1(GST-M1) genotype present increased probability of developing adverse effects and increased incidence of lymphopenia during AZA treatment. But not all scholars attribute AZA-induced cytopenia to bone marrow suppression. Azathioprine could also trigger suicidal erythrocyte death, leading to anemia. ${ }^{9}$ Much more various research is on the way to explain reasons.

Azathioprine is a double edged sword. TPMT and IPTA pharmacogenetic tests have not been put into practice as a clinical rheumatology guideline in Asia. Even patients with negative test results could have pancytopenia either, and their cost-effectiveness is unknown. The tests to everyone before access to azathioprine are unlikely available here as a developing country. Regular monitoring of blood counts (once a week during first 2 months of AZA usage and once every 1 month later when dosage is fixed)are highly recommended to reduce the possible serious myelosuppression induced by azathioprine.

\section{REFERENCES}

1. Salavaggione OE, Wang L, Wiepert M, Yee VC, Weinshilboum RM. Thiopurine S-methyltransferase pharmacogenetics: variant allele functional and comparative genomics. Pharmacogenet Genomics 2005; 15:801-15.

2. Xiao-chun WU, Hui XIONG, Lei XIONG, et al. Research on the relationship between thiopurine $\mathrm{S}$-methyltransferase genetic polymorphisms and azathioprine adverse drug reactions in patients with kidney transplantation. Chin J Clin Pharmacol Ther 2008; 13:1037-43.

3. de Boer NK, van Bodegraven AA, de Graaf P, et al. Paradoxical elevated thiopurine S-methyltransferase activity after pancytopenia during azathioprine therapy: potential influence of red blood cell age. Ther Drug Monit 2008; 30: 390-93.

4. Compagni A, Bartoli S, Buehrlen B, et al. Avoiding adverse drug reactions by pharmacogenetic testing: a systematic review of the economic evidence in the case of TPMT and AZA-induced side effects. Int J Technol Assess Health Care 2008; 24:294-302.

5. Zabala-Fernández W, Barreiro-de Acosta M, Echarri A, et al. A pharmacogenetics study of TPMT and ITPA genes detects a relationship with side effects and clinical response in patients with inflammatory bowel disease receiving Azathioprine. J Gastrointestin Liver Dis 2011; 20:247-53.

6. Yamamoto K, Okada Y, Nakamura K, et al. Inosine triphosphate pyrophosphatase $94 \mathrm{C}>\mathrm{A}$ polymorphism: clinical implications for patients with systemic lupus erythematosus treated with azathioprine. Expert Opin Drug Saf 2010; 9:44757.

7. Hawwa AF, Millership JS, Collier PS, et al. Pharmacogenomic studies of the anticancer and immunosuppressive thiopurines mercaptopurine and azathioprine. Br J Clin Pharmacol 2008; 66:517-28.

8. Stocco G, Martelossi S, Barabino A, et al. Glutathione-S-transferase genotypes and the adverse effects of azathioprine in young patients with inflammatory bowel disease. Inflamm Bowel Dis 2007; 13:57-64.

9. Geiger C, Föller M, Herrlinger KR, Lang F. Azathioprine-induced suicidal erythrocyte death. Inflamm Bowel Dis 2008; 14:1027-32. 